DOI: https://doi.org/10.14311/TPFM.2022.002

\title{
FOUNDATIONS OF ENGINEERING MATHEMATICS FOR FLUID FLOWS
}

\author{
Yuli D. Chashechkin ${ }^{1}$ \\ ${ }^{1}$ Laboratory of Fluid Mechanics, Ishlinsky Institute for Problems in Mechanics RAS, \\ prospect Vernadskogo 101/1, 119526 Moscow, Russian Federation)
}

\begin{abstract}
Based on a definition of engineering mathematics, following from Aristotle and Leibniz's philosophical principles for science, the system of fundamental equations was selected to describe fluid flow dynamics and structure. Complete solutions of the linearized system in total and abridge versions, constructed with the condition of compatibility, describe different waves and ligaments. Weak non-linear approximation describes the effect of flow components interactions and boundary conditions impact. In experiments, ligaments are manifested as sharp interfaces and fibrous. Numerical patterns of stratified flows are analyzed and compared with schlieren visualization of flow fields.
\end{abstract}

Keywords: fluid, definition, flow, energy, transport, fundamental equations, complete solution, wave, ligaments

\section{Introduction}

By recognizing mathematics as the language of science, Galileo opened a new stage in the study of nature, allowing the use of axiomatic and empirio-axiomatic approaches. Both directions began to develop actively in general mechanics and fluid mechanics. The empirio-axiomatic approach is based on Galileo's laws of inertia (equivalence of coordinate systems moving with constant velocity), and conservation of momentum by R. Descartes, energy by G. Leibniz, and matter (constituting substances).

Along with the development of a fundamental approach, constitutive models were created as well. Their development was greatly influenced by I. Newton, who formulated three laws of mechanics, including the concepts of inertia, acceleration, and force, which is non-invariant quantity determined in the coordinate system associated with the body under study only. L. Euler extended Newton's approach to the description of the fluid flow. Performing the "solidification of a liquid particle" and calculating the forces acting on a polyhedral element of a continuous medium, L. Euler constructed the first system of equations of motion for ideal fluids. C. Navier derived a system of equations for the motion of viscous fluids, taking into account the atomic hypothesis of the structure of matter of P.-S. Laplace.

Both approaches had a great influence on the development of engineering disciplines. Gradually, the development of mankind, the growth of the economy, and industry have changed the requirements for engineering sciences and added to the traditional task of operability providing and design optimization the demands to assess the long-time impact of the created technologies on the environment.

Gradually, the standards for describing and forecasting the weather, assessing climate variability, and pre-estimation of the new technologies' impact on the state of the atmosphere, ocean, taking into account both fast local and slow global phenomena, have changed. The new criteria increase the requirements for engineering disciplines in terms of objectivity and completeness of describing the dynamics and structure of flows, calculating the transfer of impurities that affect the ecology, predicting the impact of anthropogenic factors. The purpose of this work is to present the foundations of engineering mathematics to create a closed and solvable theory of flows of liquids and gases. General requirements for an adequate theory, principles of choosing basic variables and governing equations, analysis of the properties of their solutions, obtained taking into account the compatibility condition that ensures the fulfilment of the initial and boundary conditions, are discussed. Comparisons of the obtained solutions with data from laboratory experiments are given briefly.

\section{General Principles of the science philosophy}

The modern list of requirements for the logic of scientific theory, which Aristotle began to create reflecting on the nature of scientific knowledge, and continued by a number of scientists, among which Leibniz's contribution stands out, includes: 
Meaningfulness - definability of the essence of a studied subject in considered and independent categories. The basis of mechanics is formed by axiomatically defined mathematical and physical concepts of number, set, space and time, motion, matter (constituents of fluid or gas and their properties), flow;

- Identities - complete conservation of the meaning of an object at operation;

- Consistency - two opposed properties cannot be true or false simultaneously;

- Uniqueness - excluded third, from the conflicting judgments, one is true, the other is false, and the third is not given (dual logic);

- Sufficient reason - the presence of predecessors, history and independent confirmation of meaning;

- Minimum sufficiency - of all the explanations, the best is the simplest;

- Causality - state and changes are a consequence of the previous and the cause of the future;

- Completeness - description of the known properties of a subject with error estimation and the potential to describe newly discovered features.

The study of liquids and gas flows in order to guarantee the estimation of the error is carried out taking into account the above principles in the framework of Engineering mathematics, which is defined as: "An axiomatic science about the principles of choosing the content of symbols, rules of operations and criteria for assessing accuracy", and Technical physics - "An empirio-axiomatic science on the rules for choosing physical quantities, and methods for measuring and evaluating errors in the determination of their values". The common basis of both disciplines is conservation laws. On this basis, the concept of "motion" is determined in mathematics and "the state of and fluid flow" in mechanics. Common basis allows direct comparison of independently obtained theoretical and experimental results.

A special place in the definition of dual disciplines belongs to the concept of "accuracy" in the theoretical and "errors or inaccuracy" in the practical description of phenomena respectively.

In mathematics, internal criteria for assessing accuracy are naturally formulated in arithmetic and algebra of number fields on the basis of the distinguished properties of two numbers, "zero" and "one". In the mathematical analysis of continuous quantities, the analogues procedure for comparison of infinitely decreasing variations of the primary variable and functions, i.e. the Cauchy - Weierstrass procedure, is developed. In modern applied mathematics, where calculations of conditionally converging series and diverging (singular) functions are widely used, the introduction of a universal criterion at this stage is difficult and requires an individual analysis of a problem under study. One of the tools for determining the accuracy is the procedure for comparing the calculations with the experimental data, which needs for including the identity proof of the compared quantities defined in different branches of sciences. In mechanics, the conservation laws have generalized the historical experience of describing phenomena; reflect the fundamental properties of the existence and conservation of matter as well as parameters of its motion. They are based on the concept of homogeneity of space and time.

Traditionally, fluid mechanics is developed in the "continuous medium" approximation. However, the "continuous medium" methodology with continuous values of the physical quantities themselves and their derivatives on arbitrary scales does not match with the concepts of the discrete structure of matter where atomic-molecular properties are expressed on scales of the order of $10^{-8} \ldots 10^{-7} \mathrm{~cm}$ and nuclearproperties are on scales of the order of $10^{-13} \mathrm{~cm}$.

At intermediate scales of the order of $10^{-7} \ldots 10^{-6} \mathrm{~cm}$, i.e. on the size of an atomic-molecular cluster, the impact of both the atomic-molecular interactions and macroscopic properties are pronounced, for example, in the form of latent potential part of internal energy and its transformations. The choice of a description based on a scale-invariant set of conservation laws allows passing from the model of a discrete medium to a continuous one while preserving the meaning of the characterizing quantities. The sizes of the microstructural components establish the natural limits of the continuous medium model validity, namely, the minimal sizes of the studied macroscopic phenomena should exceed the scale of the molecular cluster.

The engineering sciences under consideration are based on universal conservation laws of matter (total mass or density), as well as measures of continuous medium motion, which are momentum and total energy. The mobility of atoms, molecules, and their associations - clusters or macroscopic "liquid particles", larger structural components, leads to a continuous change of the distribution of matter and the tensor of inertia. Due to independent mobility of small components changing in the moment of inertia and the inhomogeneous dissipation rate of the momentum or realizing latent internal energy, the angular momentum cannot be used as an invariant of the flow and, thus, is not considered further.

The main parameter characterizing the state and dynamics of fluid and gas is the total energy $E_{t}$, including mechanical part (kinetic and potential) and internal energy, which is determined by equilibrium 
thermodynamic parameters (Gibbs potential) [1]. Internal energy contains available potential surface, chemical, electromagnetic and other types of energy.

The total energy concept gives room to consider all the mechanisms for its transfer in a flow, including transfer with a local flow velocity and a group wave one, slow diffusion processes with a characteristic rate, and rather fast ones in the course of localized direct atomic-molecular interactions (for example, at the size of a molecular cluster upon free surface elimination in merging fluids [2].)

Transfer of invariant quantities by atomic-molecular processes is described by its own diffusivedissipative laws, characterized by own dissipative coefficients, such as, kinematic viscosity $v$, thermal diffusivity $\kappa_{T}$, and diffusion $\kappa_{S}$, for momentum, heat and substance transfer, respectively. Although the potentials characterize thermodynamically equilibrium states, their application to the description of the non-equilibrium processes is justified by small deviations of the state of systems from the equilibrium one. Large deviations from equilibrium values are described by fast-localized sources, which determine energy changes in the course of direct atomic-molecular processes on small scales.

\section{Basic mathematics of a fluid flow}

The mathematical basis for the theory of a fluid flow, describing the position, dynamic state, and physical properties of a medium, includes real or complex numbers, with the properties defined a priori. The concepts of "space" and "time" in classical mechanics were introduced axiomatically as two independent continuous sets existing independently on the nature of matter and material processes. By introducing a coordinate frame, each point in space is associated with an element from the set of real numbers. Observable invariants of the spaces, which are distances between points or time intervals between events, are used to define coordinate values. The space is a three-dimensional metric, (Euclidean), homogeneous and isotropic. An important property of a vector space is the availability of outer composition operations that is the saving of a scalar by a vector product in the initial vector space.

In the aggregate of the configuration space transformations, the orthogonal mapping into themselves (with the conservation of the distance between the elements) is distinguished. This transformation in the Cartesian coordinate system are $x_{i}^{\prime}=a_{i k} x_{k}, a_{j i} a_{j k}=0$ at; $i \neq k$; at. $a_{j i} a_{j k}=1$ at $i=k$.

The motion in a geometric sense is defined as a continuous orthogonal transformation of metric space into itself with time $t$ as an independent continuous parameter of transformation, saving the distances between points and their relative positions. In this case, the determinant composed of the coefficients of the matrix $a_{i k}$, equals $\left\|a_{i k}\right\|=+1$. An orthogonal transformation with the determinant $\left\|a_{i k}\right\|=-1$, which does not conserve the orientation of the figures, specifies a reflection about some axis. A motion in Euclidean space $\mathrm{R}^{3}$ is characterized by a group of transformations, which includes independent subgroups of rectilinear shifts and rotations. The transformations given by the group of motions are studied by elementary geometry.

The transition from geometry to mechanics includes the introduction of the concept of mass $M$, which is an additional independent quantity with its own dimension. Mass is a positively defined scalar quantity, which, hereinafter, is considered is a measure of inertia and the gravitational interaction of bodies.

The concept of motion in mechanics, which is translation of a material point, includes rectilinear shift in configuration space $\delta \mathbf{r}=\mathbf{v}_{t} \delta t$ with velocity $\mathbf{v}_{\tau}$ or rotation around the instantaneous center $\mathbf{r}=0$ with angular velocity $\boldsymbol{\Omega}$ and local velocity $\mathbf{v}=\mathbf{v}_{t}+\boldsymbol{\Omega} \times \delta \mathbf{r}$.

\section{Parameters of a fluid flow and governing equations system}

Description of substance flows in liquid, gaseous, plasma states is based on the concept of "continuous medium", which allows the use of infinitely differentiable functions over the entire range of scales. The key property of a fluid is liquidity, i.e. the ability to flow under the action of any small perturbations. This property manifests itself in the Cauchy-Helmholtz decomposition of the flow velocity of $\mathrm{v}_{i}\left(r_{r}+\delta r_{k}\right)=\mathrm{v}_{i}\left(r_{k}\right)+\varepsilon_{i j k} \Omega_{j} \delta r_{k}+\frac{\partial \mathrm{v}_{i}}{\partial x_{l}} \delta r_{l}$, where $\varepsilon_{i j k}$ is the unit antisymmetric tensor of the third rank.

The difference between the representations of "motion" and "flow" reflects the existence of two independent continuums, that are, a metric space (whose motions form a group) and an immersed medium (decomposition of a flow velocity includes the shift operator). To save independent identities for two 
spaces at the description of the flows of a continuous medium, the dimension of the physical space of a problem and of configuration space must be distinguished. The natural expansion of problem dimension is the introduction of the variable density, which corresponds to the properties of fluids. The dependence of the fluid density on other thermodynamic quantities describes the equation of state.

In modern fluid mechanics, the main parameter of an equilibrium continuous medium at rest is the free enthalpy or the Gibbs potential $G[1,2]$. Derivatives of the thermodynamic potentials define density $\rho\left(x_{1}, x_{2}, x_{3}\right)$, pressure $P\left(x_{1}, x_{2}, x_{3}\right)$, temperature $T\left(x_{1}, x_{2}, x_{3}\right)$, concentration of dissolved or suspended particles $S_{i}\left(x_{1}, x_{2}, x_{3}\right)$, which have a clear physical meaning and are available for observation [1]. Such parameters of the medium, such as density and pressure, are considered to be quantities of a double nature, namely, mechanical and thermodynamic. The equilibrium distributions of the Gibbs potential $G(\mathrm{x}, \mathrm{t})$ and all its derivatives form conventional equation of state $\rho=\rho(P, T, S)$. Besides thermodynamic potentials and their derivatives a medium which is characterized by kinetic and other physical coefficients (in particular, those determining the propagation of electromagnetic or acoustic waves), is called weakly compressible fluid, if occupying a finite volume, or gas/plasma, if filling all available space.

The main parameters a fluid flow are the specific total energy $E_{t}$ and momentum $\mathbf{p}=\rho \mathbf{v}$, which manifest themselves in the forceful action in dynamics and continuous variations in the flow structure, i.e. in evolution of distinguished spatial patterns of different physical quantities.

Definition of a fluid flow:

Forced or self-induced transfer of momentum $\mathbf{p}=\rho \mathbf{v}$, energy $E_{t}$, and matter, accompanied by selfconsistent changes in physical quantities which determine the state of a continuous medium.

Redistribution of matter and energy without transfer of momentum is called a process (for example, diffusion transport of matter).

The axiomatically introduced system of equations for fluid motion, taking into account the general principles for the choice of physical quantities and fundamental conservation laws was presented firstly in [3]. Under the assumption that the gradients of potentials, physical quantities, and the intensity of different external sources of matter $Q_{\rho}$, momentum $Q^{i}$, tempetature $Q_{T}$ and salt $Q_{S}$ are small, the basic system describes the transfer of matter, the concentration of individual components, temperature, and momentum $[2,3]$

$$
\left\{\begin{array}{l}
G=G(P, S, T)=G(\mathbf{x}, t), \rho=\rho(P, S, T)=\rho(\mathbf{x}, t), \\
\frac{\partial \rho}{d t}+\nabla_{j}\left(p^{j}\right)=Q_{\rho} \\
\frac{\partial\left(p^{i}\right)}{\partial t}+\left(\nabla_{j} \frac{p^{j}}{\rho}\right) p^{i}=-\nabla^{i} P+\rho g^{i}+\mu \Delta\left(p^{i}\right)+2 \varepsilon^{i j k} p_{j} \Omega_{k}+Q^{i}, \\
\frac{\partial \rho T}{\partial t}+\nabla_{j} \cdot\left(p^{j} T\right)=\Delta\left(\kappa_{T} \rho T\right)+Q_{T}, \\
\frac{\partial \rho S_{i}}{\partial t}+\nabla_{j} \cdot\left(p^{j} S_{i}\right)=\Delta\left(\kappa_{S} \rho S_{i}\right)+Q_{S i} .
\end{array}\right.
$$

where $\mu=\rho v$ is dynamic viscosity, $\kappa_{T}$ and $\kappa_{S}$ are coefficients of temperature and salinity diffusivites, $g$ is gravity acceleration, $\boldsymbol{\Omega}$ is global rotation angular velocity, $\nabla$ is Hamilton operator, $\varepsilon^{i j k}$ is absolutely antisymmetric unit tensor.

Connected equations (1) forming a parametrically and length scale-invariant system of coupled algebraic-differential equations is supplemented with physically based initial and boundary conditions. Consistency of the infinitesimal symmetries of the system (1) with the basic principles of physics [2] testifies to the validity of its choice as a universal basis for studying fluid flows. The complete solution of the system (1) is searched with the compatibility condition [2]. The rank of the system (the order of the highest derivative), as well as the order of its linearized version and the degree of the characteristic (dispersion) equation, determines the minimal number of eigenfunctions, which constitute a complete solution.

The fields for each independent physical quantity, which are characterized by their own geometry, spatial and temporal scales, must be simultaneously determined in the experiment. The unity of the 
content of physical parameters in theory, numerical modeling, and experiments based on system (1), gives room to estimate the accuracy of solutions. The accuracy of the state equations, which is the difference in the calculated values of density, speed of sound, refractive index, and other reliably determined quantities from (1) and obtained experimentally by measuring temperature, pressure, salinity and other quantities, determines the error of analytical and numerical calculations. System (1) contains no additional parameters and does not require their introduction for numerical codes development.

\section{Classification of infinitesimal periodic flows components}

The density of a fluid in environmental and industrial conditions $\rho(z)$ that is specified by the profiles of pressure, temperature, and concentration of impurities, under the action of buoyancy effects becomes stably stratified. Thel density profile is described by length scale $\Lambda=\left|\frac{1}{\rho} \frac{d \rho}{d z}\right|^{-1}$, frequency, $N=\sqrt{g / \Lambda}$, and period of buoyancy $T_{b}=2 \pi / N$, (the axis $z$ is directed vertically upward, $g$ is gravity acceleration, the effect of compressibility is neglected). In strongly stratified fluid, which is typical for laboratory conditions, $N \sim 1 \mathrm{~s}^{-1}$, in the environment, $N \sim 0.01 \mathrm{~s}^{-1}$, in potentially homogeneous fluid, $N \sim 10^{-5} \mathrm{~s}^{-1}$, and for actually homogeneous fluid, which is generally used in theory, $N \equiv 0$.

Taking into account the stratification effects allows to construct complete solutions of the linearized system (1) using the compatibility condition and to give a classification of the flow structural components [4]. The set of equations (1) for stratified fluids contains small dissipative coefficients and is treated by singular perturbation theory methods. Substitution of the solution in the form of plane waves with a positive frequency $\omega>0$ and a complex wave number $\mathbf{k}=\mathbf{k}_{1}+i \mathbf{k}_{2}$ for the density $\rho^{\prime}=A_{\rho} \exp i(\mathbf{k} x-\omega t)$ and similarly for perturbations of other physical quantities into the linearized system (5) gives the dispersion equation of tenth degree of multiplicative type [4]. The main regular operator with the imaginary part being small compared to the real one, describe infinitesimal waves of various types, which are inertial, gravitational, acoustic, or hybrid ones. A rich family of with singularly perturbed roots characterizes ligaments. The real and imaginary parts of these roots are in the same order.

Waves are defined as components of a flow where the parameters of local temporal variability (frequency $\omega$ ) and instantaneous spatial structure (wavenumber $\mathbf{k}$ or wavelength $\lambda$ ) are related by a dispersion relation, $\omega=\omega(\mathbf{k}, \mathbf{k A}, \ldots)$, where $\mathbf{A}$ is the amplitude.

Ligaments are thin and extended components of flows described by the set of singular solutions of the complete and linearized system (1). The number of ligaments depends on the system (1) rank. The length scales are defined by the kinematic coefficients, $v, \kappa_{T} \kappa_{S}$, as well as the wave frequency $\omega$, for periodic ligaments, $\delta_{\omega}^{v}=\sqrt{v / \omega}, \delta_{\omega}^{\kappa_{T}}=\sqrt{\kappa_{T} / \omega}, \delta_{\omega}^{\kappa_{S}}=\sqrt{\kappa_{S} / \omega}$, the time interval $\tau$ for the flow formation duration for transient ligaments, $\delta_{\tau}^{v}=\sqrt{v \cdot \tau}, \delta_{\tau}^{\kappa_{T}}=\sqrt{\kappa_{T} \cdot \tau}, \delta_{\tau}^{\kappa_{S}}=\sqrt{\kappa_{S} \cdot \tau}$, and the stationary flow with velocity $U, \delta_{U}^{v}=v / U, \delta_{U}^{\kappa_{T}}=\kappa_{T} / U, \delta_{U}^{\kappa_{S}}=\kappa_{S} / U$. The length of ligaments depends on the lifetime of a process under study. Ligaments are distinguished by a high level of vorticity and mechanical energy dissipation rate. All the flow components co-exist, transfer, and disappear simultaneously, despite the difference in characteristic scales. A consequence of the high rank of the system of fundamental equations is that all flows described by a set of regular and singular functions, in the general case, by incommensurable parameters, are unsteady. In some experiments, ligaments cannot be identified due to the low sensitivity or resolution of instruments and shadowing by large-scale high-level perturbations.

Using all solutions for the system (1) and the dispersion equation allows solving the linear problem on periodic internal wave generation by an oscillating body in complete $2 \mathrm{D}$ and $3 \mathrm{D}$ formulations with physically justified initial and boundary conditions [5].

Calculating the ligaments generated by periodic internal waves incident on an inclined wall or a critical level, at which the wave and buoyancy frequencies coincide in a medium with a variable density gradient, gives room to completely solve the wave problem. The differences between the calculations of evanescent waves infiltrating into the supercritical region, where their frequency exceeds the local buoyancy frequency, which was published in 1998 [6], from the results of experiments [7] published in 2012, do not exceed a few percent. The analysis of solutions to problems of joint generation of internal 
waves and ligaments in the approximation of weak nonlinearity of equations and boundary conditions was carried out in [8].

\section{Studies of flows around an obstacle based on a truncated fundamental system}

Noticeable the stratification effects on the dynamics and geometry of flows and are actively studied. Practically differential heating and profiling of the stratifying salinity are used. Due to the smallness of the ratio of kinetic coefficients (both for seawater and an aqueous solution of common table salt, the Lewis number is $\mathrm{Le}=\kappa_{T} / \kappa_{S} \sim 100$ ), salt stratification decay more slowly than temperature one, and is used widely [2]. A fluid flow is considered isothermal and incompressible with the state equation defined by the profile of the dissolved salt concentration $\rho_{0}(z)=\rho_{00} S_{0}(z)=\rho_{00} \exp (-z / \Lambda)$ with constant values of buoyancy scale $\Lambda$, frequency $N$, and period $T_{b}$.where ( $\rho_{00}$ is the density on the reference level). The system of fundamental equations (1) for a one-component incompressible stratified medium in the Boussinesq approximation, takes the form,

$$
\begin{aligned}
& \rho=\rho_{0}+\rho_{00} \cdot s, \quad \operatorname{div} \mathbf{v}=0 \\
& \frac{\partial \mathbf{v}}{\partial t}+\nabla \cdot(\mathbf{v} \mathbf{v})=-\frac{1}{\rho_{00}} \nabla P+\nabla \cdot(v \nabla \mathbf{v})-s \cdot \mathbf{g}, \\
& \frac{\partial s}{\partial t}+\nabla \cdot(s \mathbf{v})=\nabla \cdot\left(\kappa_{S} \nabla s\right)+\frac{\mathbf{v}_{z}}{\Lambda},
\end{aligned}
$$

where fluid velocity is $\mathbf{v}=\mathbf{p} / \rho, P(x, z, t)$ is the pressure except for the hydrostatic one, $S=S_{0}+s, s$ is the salinity perturbation including the salt contraction coefficient.

No-slip and no-flux boundary conditions on the surface of a solid body with of length $L$, width $W$, and height $h$, which can move with a constant velocity $U$ starting at $t=0$, have the following form

$\left.\mathbf{u}\right|_{t \leq 0}=0,\left.\quad s\right|_{t \leq 0}=0,\left.P\right|_{t \leq 0}=0,\left.\mathrm{u}_{x}\right|_{\Sigma}=\left.\mathrm{u}_{z}\right|_{\Sigma}=0, \quad\left[\frac{\partial s}{\partial \mathbf{n}}\right]_{\Sigma}=\frac{1}{\Lambda} \frac{\partial z}{\partial \mathbf{n}},\left.\mathrm{u}_{x}\right|_{x, z \rightarrow \infty}=U,\left.\quad \mathrm{u}_{z}\right|_{x, z \rightarrow \infty}=0$.

The governing system of equations (2) together with the initial and boundary conditions (3) are characterized the buoyancy scale, $\Lambda=|d \ln \rho / d z|^{-1}$, the geometric dimensions of the body, $h, L, W$, attached internal wave length $\lambda_{a}=U T_{b}$, and viscous wave scale $L_{v}=\sqrt[3]{g v} / N$. Small scales characterizing basic ligaments accompanying stratification and the basic flow with velocity $U$ are $\delta_{N}^{v}=\sqrt{\nu / N}, \delta_{N}^{\kappa_{S}}=\sqrt{\kappa_{S} / N}$, and $\delta_{U}^{v}=v / U, \delta_{U}^{\kappa_{S}}=\kappa_{S} / U$, respectively. The system (2, 3) was selected as a basis for numerical and laboratory studies of flows around an obstacle.

The comparisons of the numerical and schlieren visualization data are presented in Fig. 1. Here, the objects of comparison are the components of the density gradient fields, calculated theoretically and reconstructed from the schlieren visualization data of flows of an aqueous solution of table salt [9].
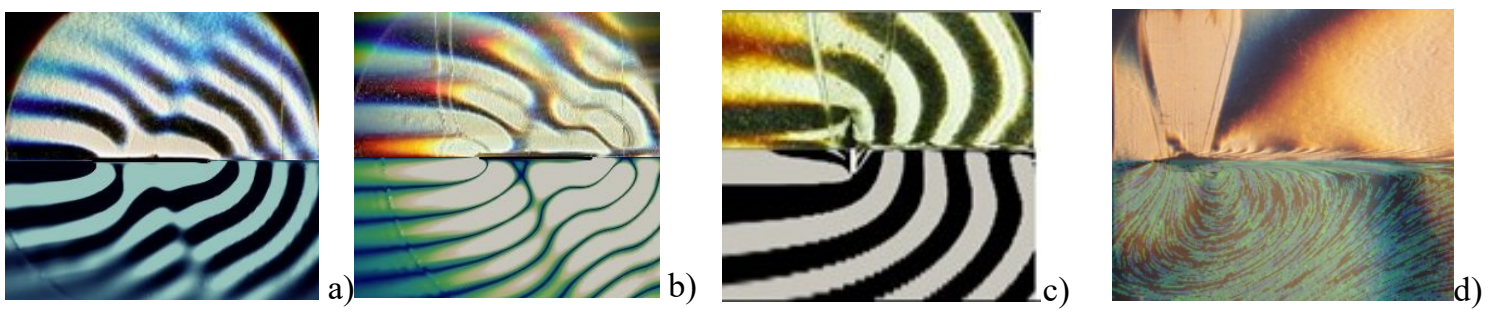

Figure 1: Schlieren (upper part of the images) and calculated (lower one) stratified flow patterns around a uniformly moving horizontal plate, $\left.T_{b}=7.6 \mathrm{~s},: a-c\right)-L=7.5 \mathrm{~cm}, U=0.27,0.39,0.41 \mathrm{~cm} / \mathrm{s}$; thin wavy lines in the Schlieren images are density markers that visualize the profile of the upstream horizontal velocity component; $d$ ) $-L=2.5 \mathrm{~cm}, U=2.3 \mathrm{~cm} / \mathrm{s}$, lower part presents calculated streamline pattern

The density and refractive index of the working fluid are related by an almost constant coefficient. When the internal wave length $\lambda=U T_{b}$ is less than the plate length $(\lambda<L)$. the phase surfaces are 
broken above and beneath the plate (Fig. 1, a, b). Increase in the velocity changes in the geometry of the fine-structural interfaces, and manifestation degree of separate flow components (Fig. 1,c). The strongest structural changes are revealed in the wake past the plate, where a system of short interfaces in the form of tilted ligaments (streaky structure) is observed (Fig. 1, d).

In the vortex flow regime, when the vortex elements become a dominant flow component with the internal wavelength being comparable to the observation area size, the most contrast structural changes are manifested in the wake flow past the plate (Fig. 2). Both laboratory and numerical simulations show that the wake flow structure past the tilted plate consists of a typical vortex street. In the strongly stratified medium (Fig. 2, a), the wake vortices gradually collapse downstream transforming into a set of fineelements, while in the homogeneous fluid (Fig. 2,b), the vortex street expands in the vertical direction. A variety of multilayer fine-structural flow elements is formed on the vortex.
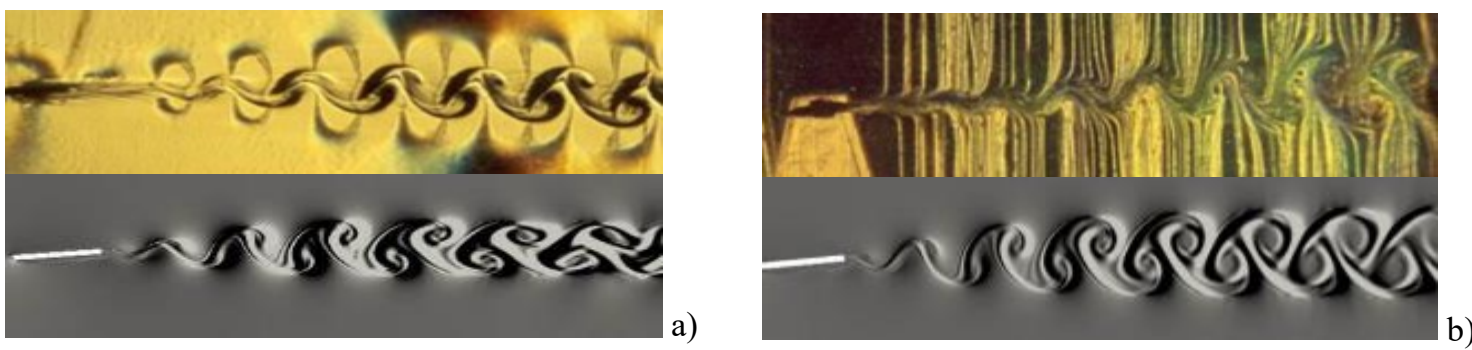

Figure 2: Schlieren (upper part of the images) and calculated (lower one) vortex flow patterns past a uniformly moving tilted plate $(L=2.5 \mathrm{~cm}, U=4.3 \mathrm{~cm} / \mathrm{s}): \mathrm{a}, \mathrm{b})-T_{b}=7.6 ; 6.3 \cdot 10^{5} \mathrm{~s}, \quad N=0.83 ; 10^{-5} \mathrm{~s}^{-1}$

In the unsteady flow regime, one can distinguish slowly evolving components, such as upstream and attached wave fields, rapidly changing ones, including fine-structured layers or ligaments, and their sets forming vortices. All the flow components evolve and actively interact with each other and with the free stream. The calculations and observations of the flow are in good qualitative agreement [9].

The fine structure of flow patterns, which is a consequence of atomic-molecular interactions in a moving matter and intense action of energy transformations in the atomic-molecular processes at the boundary of fluid (gas) with a submerged solid body. As an illustration, Fig. 3 presents the schlieren visualization data of the flow pattern around the wing in the wind tunnel (photographs were kindly provided by Professor V.G. Sudakov, TsAGI) and the plate towed in the stratified tank (Fig. 3, b, d). Families of transversely located fine-interfaces or ligaments are visualized both in compressible gas flows with transonic velocities, and near a slowly moving body in a weakly compressible stratified fluid.

The presented results of theoretical and experimental studies of the flow pattern around the strip show that the reduced system of fundamental equations (2) with boundary conditions (3) describes all the details of the observed flow pattern, which are upstream disturbances, internal waves, wake, vortices, ligaments, in a wide range of flow parameters.
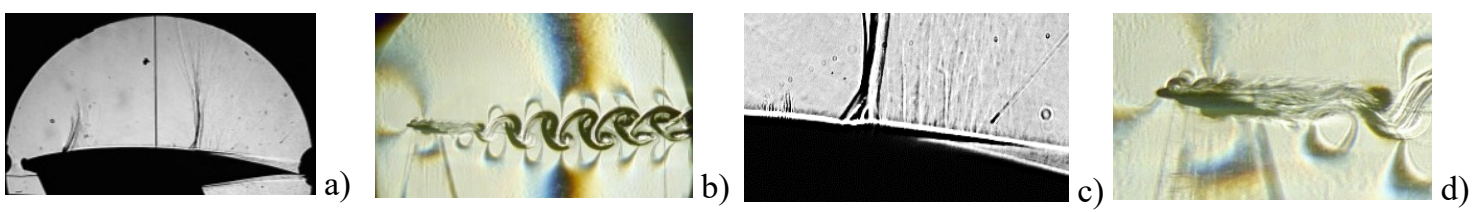

Figure 3: Schlieren images of flow: $a, c)$ - wing in the TsAGI wind tunnel at $\mathrm{Ma}=0.77$ and plate in the stratified basin of the IPMech RAS $\left(T_{b}=7.55 \mathrm{~s}, L=2.5 \mathrm{~cm}\right.$, angle of attack $\alpha=12.5^{\circ}, U=3.6 \mathrm{~cm} / \mathrm{s}$, $\operatorname{Re}=U L / v=900, \operatorname{Fr}=U / N L=1.73) ; b, d)$ - enlarged sections of the figures with fine structures

At high velocities, the flow pattern becomes more complicated, and the flow, which contains a large number of structural components with its own parameters, is continuously transformed [9]. Algorithms and criteria for constructing complete solutions of equations $(2,3)$ allow calculating all the physical quantities of flows. The fields of density, velocity, pressure, vorticity, its baroclinic generation rate, mechanical energy dissipation rate, forces, and torques acting on a 2D body in a flow, were calculated without involving additional hypotheses and constants both in stratified and homogeneous (potentially and actual) environments [9]. The transition to a 3D formulation requires the development of new 
algorithms, which take into account the dimension expansion of the complete space of a problem and the appearance of new groups of ligaments. In this case, solving problems in a 2D formulation can be used to control the quality of the developed algorithms and methods for comparing theoretical and experimental results.

Selecting the variables of the problem such as are density, momentum, pressure, the concentration of the stratifying component, chosen in accordance with the conservation laws, the principles of accuracy (error) control can be implemented by directly comparing the observed and calculated fields. The same physical quantities obtained by independent methods, including analytical one (in this case, non-uniform asymptotic expansions do not allow an estimation of accuracy), numerical one, obtained taking into account the solvability condition of ligaments, and experimental one with a high spatial resolution.

\section{Conclusion}

Using principles of Aristoteles and Leibnitz logics the list requirements for constructions of a consistent mathematical model and organization of complete experiment are proposed.

Concepts of "motion" as a continuous transformation of 3D metric space into itself and "fluid flow" as transfer of momentum, energy, and substance supplemented by consistent variations of physical quantities are distinguished.

Sets of complete solutions of the 2D system of fundamental equations describing waves, ligaments, vortices, jets, and wakes are in good agreement with consistent laboratory studies of the dynamics and structures of stratified and homogeneous fluids.

\section{Acknowledgements}

The work was carried out on the topic of a state assignment (state registration No. AAAA-A20120011690131-7).

\section{References}

[1] Feistel, R.: Thermodynamic properties of seawater, ice and humid air: TEOS-10, before and beyond. Ocean Science discussions, 2018, 14, 471-502. DOI: 10.5194/os-2018-19

[2] Chashechkin, Yu.D.: Foundations of engineering mathematics applied for fluid flows. Axioms, vol. 10: (2021) 286. https://doi.org/10.3390/axioms10040286.

[3] Landau, L.D.; Lifshitz, E.M.: Fluid Mechanics. V.6. Course of Theoretical Physics, Pergamon Press: Oxford, UK: (1987) 560 p.

[4] Chashechkin Yuli D.: Differential fluid mechanics: instrument for consistent analytical, and laboratory studies of flows. In Proceedings Topical Problems of Fluid Mechanics 2017, Prague. Edited by David Šimurda and Tomáš Bodnár: (2017) pp. 65-78. DOI: https://doi.org/10.14311/TPFM.2017.010.

[5] Vasiliev, A.Y.; Chashechkin, Yu.D.: Generation of beams of three-dimensional periodic internal waves by sources of various types. J. Appl. Mech. Tech. Phys., 2006, 47(3), 314-323.

[6] Kistovich, Yu.V.; Chashechkin, Yu.D.: Linear theory of beams internal wave propagation an arbitrarily stratified liquid. J. Appl. Mech. Tech. Phys. 1998, 39(5), 302-309.

[7] Paoletti, M. S.; Swinney, H.L.: Propagating and evanescent internal waves in a deep ocean model. J. Fluid Mech, 2012, 706, 571-583.

[8] Chashechkin, Yuli D.: Conventional partial and new complete solutions of the fundamental equations of fluid mechanics in the problem of periodic internal waves with accompanying ligaments generation. Mathematics, 2021. V. 9. No. 586. https://doi.org/10.3390/math9060586.

[9] Chashechkin Yu. D., Zagumennyi I. V.: 2D hydrodynamics of a plate: from creeping flow to transient vortex regimes. Fluids, 2021. V.6. 310. https://doi.org/10.3390/fluids6090310. 